\title{
Gemcitabine, oxaliplatin and 5-FU in advanced bile duct and gallbladder carcinoma: two parallel, multicentre phase-II trials
}

\author{
AD Wagner, ${ }^{* 1,8}$, P Buechner-Steudel', M Moehler², H Schmalenberg ${ }^{3}$, R Behrens $^{4}$, J Fahlke ${ }^{5}$, A Wein ${ }^{6}$, \\ S Behl', O Kuss', G Kleber', and WE Fleig',10
}

'First Department of Medicine, University Hospital, Halle 06097 (Saale), Germany; ${ }^{2}$ First Department of Medicine, University Hospital, Mainz 5513 I, Germany; ${ }^{3}$ Department of Medicine II, University Hospital, Jena 07740, Germany; ${ }^{4}$ Gastroenterology Practice, Halle 06108 (Saale), Germany;

${ }^{5}$ Department of Visceral Surgery, University Hospital, Magdeburg 39 I 20, Germany; ${ }^{6}$ First Department of Medicine, University Hospital, Erlangen 91054,

Germany; ${ }^{7}$ Institute of Medical Epidemiology, Biostatistics, and Informatics, Martin-Luther-University Halle-Wittenberg, Halle 06097 (Saale), Germany

BACKGROUND: Gemcitabine, oxaliplatin and 5-fluorouracil (5-FU) are active in biliary tract cancer and have a potentially synergistic mode of action and non-overlapping toxicity. The objective of these trials was to determine response, survival and toxicity separately in patients with bile duct cancer (BDC) and gallbladder cancer (GBC) treated with gemcitabine/oxaliplatin/5-FU chemotherapy. METHODS: Eligible patients with histologically proven, advanced or metastatic BDC $(n=37)$ or GBC $(n=35)$ were treated with gemcitabine $\left(900 \mathrm{mg} \mathrm{m}^{-2}\right.$ over $\left.30 \mathrm{~min}\right)$, oxaliplatin $\left(65 \mathrm{mg} \mathrm{m}^{-2}\right)$ and 5 -FU $\left(1500 \mathrm{mg} \mathrm{m}^{-2}\right.$ over $\left.24 \mathrm{~h}\right)$ on days I and 8 of a 21 -day cycle. Tumour response was the primary outcome measure.

RESULTS: Response rates were 19\% (95\% Cl: 6-32\%) and 23\% (95\% Cl: 9-37\%) for BDC and GBC, respectively. Median survivals were 10.0 months (95\% Cl: 8.6-12.4) and 9.9 months (95\% Cl: $7.5-12.2)$ for BDC and GBC, respectively, and I- and 2-year survival rates were 40 and 23\% in BDC and 34 and $6 \%$ in GBC (intention-to-treat analysis). Major grade III and IV adverse events were neutropenia, thrombocytopenia, elevated bilirubin and anorexia.

CONCLUSION: Triple-drug chemotherapy achieves comparable results for response and survival to previously reported regimens, but with more toxicity.

British Journal of Cancer (2009) I 0 I, I846- |852. doi:I0.1038/sj.bjc.6605377 www.bjcancer.com

Published online 10 November 2009

(c) 2009 Cancer Research UK

Keywords: bile duct cancer; gallbladder cancer; gemcitabine; oxaliplatin; 5-FU

Although biliary tract cancer (BTC) is a rare cancer in Europe and North America, there is substantial geographical variation, with high mortality rates in Central and Eastern Europe, Japan, India, Korea and Shanghai (China). The highest rates of up to 16.6/ 100000 women are seen in Chile (Randi et al, 2009). Biliary tract cancer includes gallbladder cancer (GBC) and bile duct cancer (BDC). Bile duct cancer may be further subdivided into intrahepatic cholangiocarcinoma and adenocarcinoma of the extrahepatic bile ducts, which includes Klatskin's tumours at the liver hilus. Unfortunately, fewer than $10 \%$ of all patients are

*Correspondence: Dr AD Wagner, Multidisciplinary Oncology Center, Lausanne University Hospital, Rue du Bugnon 46, Lausanne I0II, Switzerland; E-mail: Dorothea.wagner@chuv.ch

These studies have been presented in part at Annual Meetings of the American Society of Clinical Oncology (ASCO 2005, abstract no. 4147 and ASCO 2006, abstract no. 4I29)

${ }^{8}$ Current address: Multidisciplinary Oncology Center, Lausanne I0। I, Switzerland

${ }^{9}$ Current address: Ostalb-Klinikum, Aalen 73430, Germany

${ }^{10}$ Current address: University of Leipzig Hospitals and Clinics, Leipzig 04103, Germany

Received 28 May 2009; revised 25 September 2009; accepted 25 September 2009; published online 10 November 2009 candidates for curative resection, and relapse rates remain high after surgery (Hezel and Zhu, 2008). Thus, systemic chemotherapy is given to the majority of patients.

Compared with best supportive care, fluoropyrimidine-based combination chemotherapy has been shown in a randomised trial to improve survival and quality of life (QoL) in bilio-pancreatic cancer (Glimelius et al, 1996).

Bile duct cancer is a heterogeneous group with increasing evidence of differences between BDC and GBC at both clinical (Yonemoto et al, 2007) and molecular levels (Jarnagin et al, 2006). A recently published, pooled analysis of 104 chemotherapy trials in BTC identified response rates of 34.4 and $20.2 \%$, respectively, in patients with GBC and BDC (Eckel and Schmid, 2007). Despite the significantly better response rate in GBC, patients with GBC had a shorter median overall survival (7.2 months) than those with BDC (9.3 months; $P=0.048)$. This observation was confirmed by a large $(n=413)$, retrospective analysis from Japan reporting a median survival of 8.4 months (95\% CI: $5.5-11.2$ ) for intrahepatic cholangiocarcinoma, 10.2 months (95\% CI: 5.4-13.7) for extrahepatic BDC and 6.5 months (95\% CI: 5.3-8.0) for GBC (Yonemoto et al, 2007). In addition, several individual trials including patients with both BDC and GBC have reported clinically important differences between $\mathrm{BDC}$ and GBC, ranging from 2.7 to 12.4 months (Sanz-Altamira et al, 1998; Patt et al, 2001; Alberts et al, 2005; Knox et al, 2005; Kobayashi et al, 2006) in 
median overall survival, with constantly better results for BDC patients. In a randomised phase II trial, which compared a combination of 5-fluorouracil (5-FU)/cisplatin chemotherapy with single-agent 5-FU chemotherapy in BTC, the response rates and median overall survival tended to be superior with the doublet (18.5 vs $7.1 \%$ and 8.0 vs 5.0 months, respectively); however, the differences were not statistically significant (Ducreux et al, 2005). Significantly better response rates $(24 v s 15 \%)$ and survival (8.3 vs 11.7 months, $P=0.002$ ) for the gemcitabine/cisplatin combination - as compared with single-agent gemcitabine - have been reported in the recently presented landmark trial $\mathrm{UK}$ ABC-02, which is the largest randomised study in BTC to date (Valle et al, 2009). Although results from randomised trials comparing the combination of gemcitabine and 5-FU in BTC are lacking, the available data for the combination of gemcitabine and fluoropyrimidines in pancreatic cancer are controversial. Although the gemcitabine/5FU combination has no advantage in survival when compared with gemcitabine alone (Riess et al, 2005), a randomised phase III trial comparing gemcitabine/capecitabine $v s$ gemcitabine alone showed a significant survival benefit for the two-drug combination (HR for survival $0.8 ; 95 \% \mathrm{CI}: 0.65-0.98 ; P=0.026$ ) (Cunningham et al, 2005). Whether treatment results for biliary cancer might be further improved using three-drug combinations is still unclear. On the basis of the evidence of their activity in BTC (Eckel and Schmid, 2007), their non-overlapping toxicity profiles and a potentially synergistic mode of action (Peters et al, 1995; Faivre et al, 1999), we investigated the efficacy and safety of the triplet combination of gemcitabine, oxaliplatin and infusional 5-FU (GemFOx) in patients with locally advanced or metastatic BDC and GBC.

Separation of the patients into two parallel but separate phase II trials for BDC and GBC was motivated by both, their different prognosis and potentially different response to chemotherapy.

\section{PATIENTS AND METHODS}

\section{Eligibility criteria}

Eligible patients met the following criteria: histologically confirmed, unresectable, locally advanced (UICC stage III, $\mathrm{T}_{1-4}$ and $\mathrm{N}_{1}$ ), metastatic (UICC stage IV, $\mathrm{T}_{1-4}, \mathrm{~N}_{1}$ and $\mathrm{M}_{1}$ ) or recurring (after resection) adenocarcinoma of the bile ducts (BDC study) or gallbladder (GBC study); bidimensionally measurable tumour lesion; age 18-75 years; ECOG status 0-1; estimated life expectancy of $\geqslant 3$ months; and adequate bone marrow function (white blood cell count $\geqslant 3.5 \times 10^{9} / 1$, platelets $\geqslant 100 \times 10^{9} / 1$ ). Adjuvant chemotherapy or radiation therapy was permitted if it had been terminated at least 6 months before the inclusion in this trial. Exclusion criteria were borderline tumours, serious comorbid conditions, bilirubin $\geqslant 1.5$ times the upper limit of normal (ULN) despite adequate endoscopic biliary drainage, alanine amino transaminase (ALT) and aspartate amino transaminase (AST) levels $>5$ and 2.5 times, respectively, the ULN in patients with and without liver metastases, creatinine exceeding $200 \mu \mathrm{moll}^{-1}$, pregnancy and lactation, earlier chemotherapy, suspected cerebral metastases and peripheral sensory neuropathy. The studies were conducted in accordance with the Declaration of Helsinki Principles and Good Clinical Practice guidelines. Supportive care measures such as antibiotic therapy and administration of haematopoietic growth factors were permitted and used at the discretion of the investigator. Written informed consent was obtained from all patients before entering the study. The Ethics Committee of the Medical Faculty (Martin-LutherUniversity Halle-Wittenberg, Germany) and the responsible ethics committees for the participating institutions had approved the protocol.

\section{Study design and treatment}

These single-arm, multicentre phase II studies were conducted in parallel at the same institutions. The study protocol was approved and supported by the Arbeitsgemeinschaft Internistische Onkologie (AIO). Similar to our parallel phase ${ }^{-}$II study in pancreatic cancer (Wagner et al, 2007), patients were treated with gemcitabine ( $900 \mathrm{mg} \mathrm{m}^{-2}$ as a 30 -min infusion), followed by oxaliplatin $\left(65 \mathrm{mg} \mathrm{m}^{-2}\right.$ as a $2-\mathrm{h}$ infusion $)$ and 5 -FU $\left(1500 \mathrm{mg} \mathrm{m}^{-2}\right.$ without folinic acid as a 24 -h continuous infusion), all on days 1 and 8 of a 21-day schedule. Treatment was terminated on disease progression or unacceptable toxicity, or at the patient's or investigator's request. Drug doses were modified on the basis of blood counts taken before each administration to maintain a tolerable safety profile. Treatment was interrupted as soon as leukopenia or diarrhoea of $>$ grade I or any other toxicity (except alopecia) of $>$ grade II was observed. If time to recovery from any toxicity was more than 1 week, subsequent doses were reduced to $80 \%$. In case of sensory neuropathy lasting longer than 7 days, oxaliplatin was administered at $80 \%$ of the initial dose in subsequent cycles. When functional impairment attributed to sensory neuropathy was not relieved at the time of the next scheduled administration, treatment with oxaliplatin was withheld until recovery.

\section{Assessment}

Pretreatment evaluations consisted of a complete medical history, physical examination, assessment of ECOG status, blood count, blood chemistry including CA 19-9 and urine analysis. Tumour measurements were performed with either computerised tomography or magnetic resonance imaging every 6 weeks, with tumour responses classified according to the WHO criteria (Miller et al, 1981). Blood counts were obtained before each chemotherapy administration, and serum chemistry and urine analysis were carried out before each new cycle. Toxicity was classified according to the NCI-CTC (version 2.0). Quality of life was assessed before therapy and after every two cycles using the FACTHep questionnaire (Heffernan et al, 2002). After discontinuation of treatment, follow-up was scheduled every 3 months.

\section{Statistical methods and analysis}

According to the study protocol, three populations for analysis were defined as follows:

1. Safety population: All patients who received the study medication at least once.

2. Intention-to-treat (ITT) population: All eligible patients who received the study medication at least once.

3. Per-protocol (PP) population: All patients who completed at least two cycles of chemotherapy.

Objective response rate as the primary end point of both studies was assessed in both the PP and ITT populations. The secondary end points - median overall survival, time to progression (TTP) and 1- and 2-year overall survival rates - were analysed in the ITT population only. On the basis of the results published for singleagent therapy with gemcitabine (Valle et al, 2009), we predefined overall response rates of 10 and $30 \%$ in the PP population as being clinically irrelevant and relevant, respectively. The sample size necessary for the primary end point was calculated accepting a type I error of $5 \%$ and a test power of $90 \%$. This resulted in a target enrolment of 35 response-evaluable patients in each study. Time to progression and survival were analysed using standard methods of survival analysis. Survival function was estimated by the KaplanMeier method (Kaplan and Meier, 1958). Response rates, median survival times and survival rates are given with $95 \%$ CIs. 


\section{RESULTS}

\section{Patients and treatment}

A total of 38 patients with BDC and 37 patients with GBC were enrolled between February 2002 and October 2004. Follow-up was performed until death or up to a maximum of 60 months. Patient flow is depicted in Figure 1. Baseline demographic and clinical characteristics of the ITT and PP populations are summarised in Table 1. In the BDC study, a total of 299 cycles of study therapy were started and 280 cycles completed. The median number of cycles per patient was 6.0 (range: $1-28$ ). In the GBC study, a total of 274 treatment cycles were started and 265 completed. The median number of cycles per patient was 8 (range: $0-18$ ).

\section{Efficacy}

Objective response rates in both the PP and ITT populations are presented in Table 2. No patient in either study showed a complete response and none of the patients with a partial response qualified for secondary resection. The Kaplan-Meier curves for overall survival of the ITT populations in both trials are depicted in Figure 2.

$B D C$ trial On ITT analysis, median overall survival and time to progression were 10.0 (95\% CI: 8.6-12.4) and 6.2 (95\% CI:

Bile duct cancer study

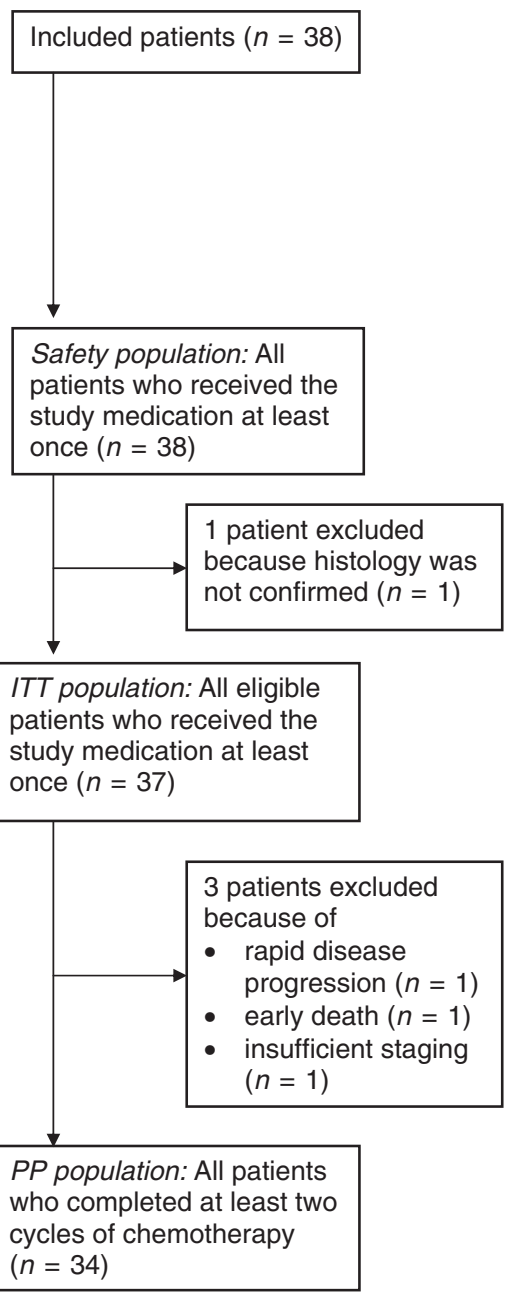

5.1-11.5) months, respectively. One- and 2-year overall survival rates were $40 \%$ (95\% CI: $25-56 \%$ ) and $23 \%$ (95\% CI: $11-38 \%$ ), respectively.

GBC trial On ITT analysis, median overall survival and time to progression were 9.9 (95\% CI: $7.5-12.2)$ and 5.7 (95\% CI: $3.1-8.1)$ months, respectively. One- and 2-year overall survival rates were $34 \%$ (95\% CI: $20-51 \%$ ) and 6\% (95\% CI: $1-16 \%$ ), respectively.

The pooled results for QoL from both studies, as measured by the FACT-Hep questionnaire, are depicted in Figure 3. Overall, the FACT-Hep global score in those patients who completed the questionnaires remained stable over the treatment period. However, these data should be interpreted with great caution because of a significant number of missing questionnaires. Bias attributed to the selective return of questionnaires from patients in good performance status cannot be excluded for this reason.

\section{Adverse events}

The most common adverse events, rated by the attending physician as NCI-CTC grade I-IV, are reported in Table 3. Treatment was well tolerated in the majority of patients. Most patients in both trials discontinued study therapy because of disease progression or deteriorating performance status. There were no treatment-related deaths.

\section{Gallbladder cancer study}
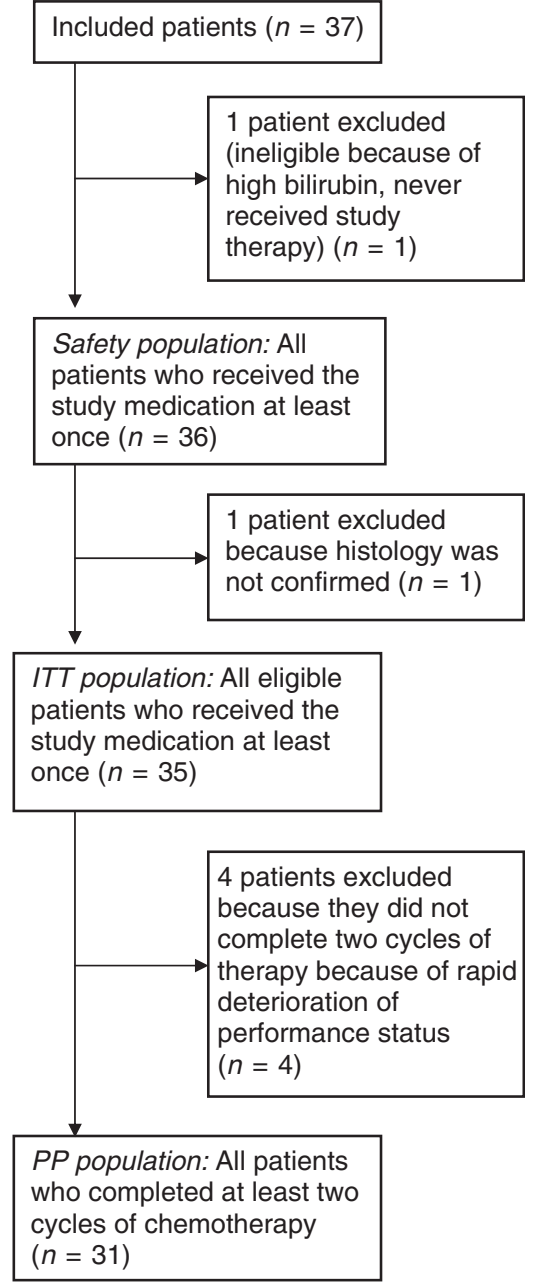

Figure I Diagram of patient flow. 
GemFOx in advanced bile duct and gallbladder cancer

Table I Baseline characteristics

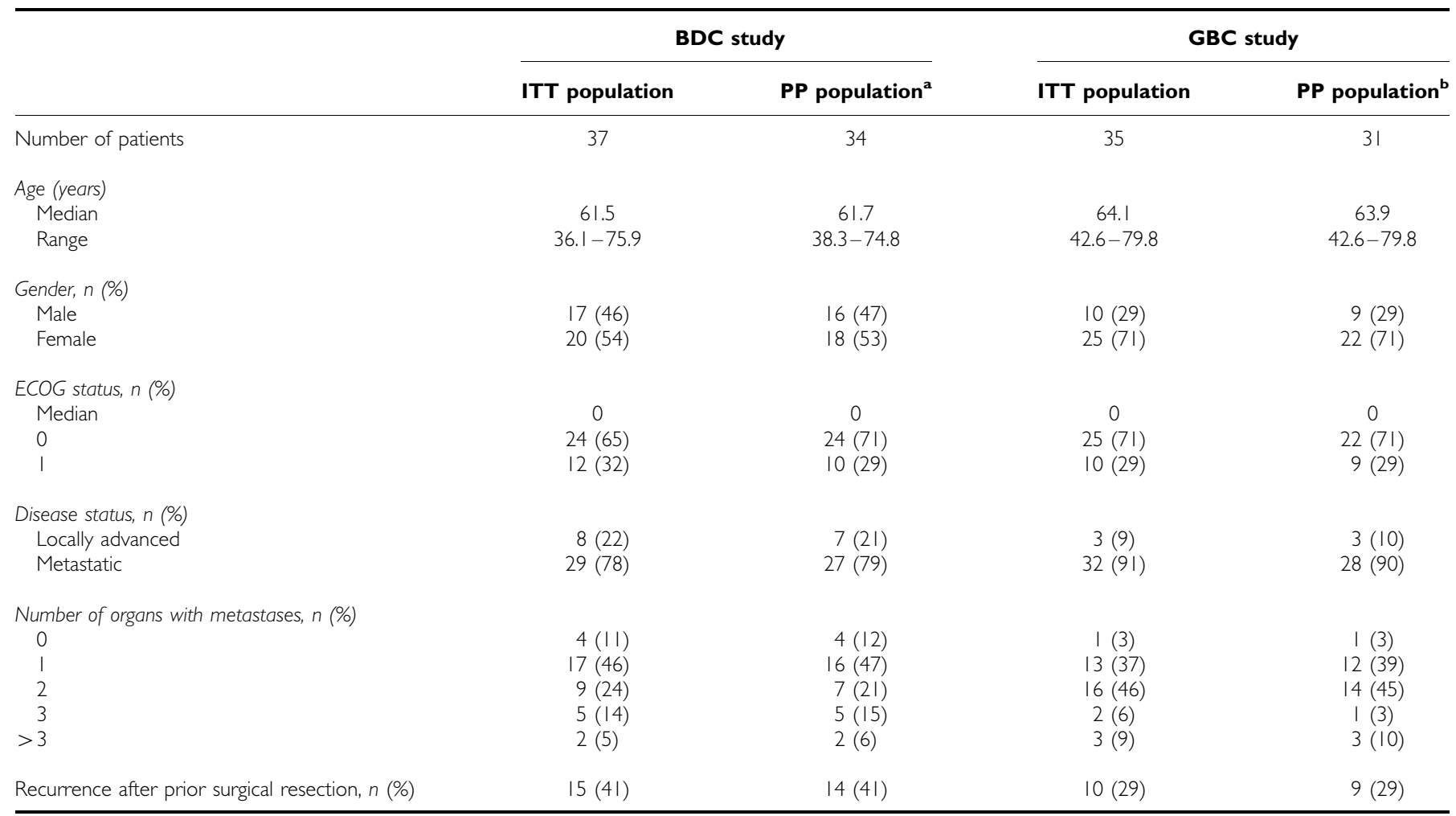

Abbreviations: $\mathrm{BDC}=$ bile duct cancer; $\mathrm{GBC}=$ gallbladder cancer; ITT population = intention-to-treat population; PP population = per-protocol population. The definitions of both populations are provided in the section 'Statistical methods and analysis'. The patient flow in both populations is depicted in Figure I. ${ }^{\text {aTwo }}$ patients were not eligible for the assessment of response according to the protocol because they did not complete two cycles of therapy. One more patient could not be assigned a response category because of insufficient staging. ${ }^{b}$ Four patients were not eligible for the assessment of response because they did not complete two cycles of therapy due to rapid deterioration of their functional status. Results for the ITT analysis are provided in the text.

Table 2 Tumour response (WHO) (Miller et al, 198I) (ITT and PP populations)

\begin{tabular}{|c|c|c|c|c|}
\hline & \multicolumn{2}{|c|}{ BDC study } & \multicolumn{2}{|c|}{ GBC study } \\
\hline & $\begin{array}{c}\text { ITT population } \\
\text { Number }(\%) \text { of evaluable } \\
\text { patients }(n=37)\end{array}$ & $\begin{array}{c}\text { PP population } \\
\text { Number }(\%) \text { of evaluable } \\
\text { patients }(n=34)\end{array}$ & $\begin{array}{c}\text { ITT population } \\
\text { Number }(\%) \text { of evaluable } \\
\text { patients }(n=35)\end{array}$ & $\begin{array}{c}\text { PP population } \\
\text { Number }(\%) \text { of evaluable } \\
\text { patients }(n=31)\end{array}$ \\
\hline CR & $0(0)$ & $0(0)$ & $0(0)$ & $0(0)$ \\
\hline PR & $7(19)$ & $7(20)$ & $8(23)$ & $8(26)$ \\
\hline NC & $20(54)$ & $20(59)$ & $16(46)$ & $16(52)$ \\
\hline Not assessable & $4(11)$ & I (3) & $5(14)$ & I (3) \\
\hline Overall response (CR+PR) & $7(19)$ & $7(20)$ & $8(23)$ & $8(26)$ \\
\hline Disease control $(\mathrm{CR}+\mathrm{PR}+\mathrm{NC})$ & $27(73)$ & $27(79)$ & $24(69)$ & $24(77)$ \\
\hline
\end{tabular}

Abbreviations: $\mathrm{BDC}=$ bile duct cancer; $\mathrm{CR}=$ complete response; $\mathrm{GBC}=$ gallbladder cancer; ITT population = intention-to-treat-population; $\mathrm{NC}=$ no change; $\mathrm{PD}=$ progressive disease; PR = partial response; PP population = per-protocol-population (both populations have been defined in the text; see 'Statistical methods and analysis' section).

$B D C$ trial A total of 25 serious adverse events occurred; of these, 12 were obstructive jaundice requiring endoscopic treatment. One patient each experienced deep venous thrombosis and pulmonary embolism. All serious adverse events except one were regarded as unrelated to therapy by the investigators. One patient with a history of cardiomyopathy suffered sudden death 1 day after the administration of chemotherapy. Whether this event was related to the underlying disease, 5-FU-associated cardio toxicity or both is not clear. A total of 21 hospitalisations were necessary in BDC patients; among these, 9 were attributable to febrile infections, mostly cholangitis (8 patients). One patient in this group had febrile neutropenia.
Chemotherapy was administered as planned for $55.3 \%$ of scheduled treatments. Dosage had to be reduced for $20 \%$ or treatment deferred for $15 \%$, or both for $7.8 \%$, of scheduled administrations. In one patient, therapy was discontinued because of an allergic reaction to oxaliplatin.

GBC trial A total of 19 serious adverse events occurred. Only one of these (dysphagia and sustained vomiting with onset soon after the administration of chemotherapy) was considered as possibly related to the study therapy by the investigator. Most other serious adverse events were rated as associated with the underlying 


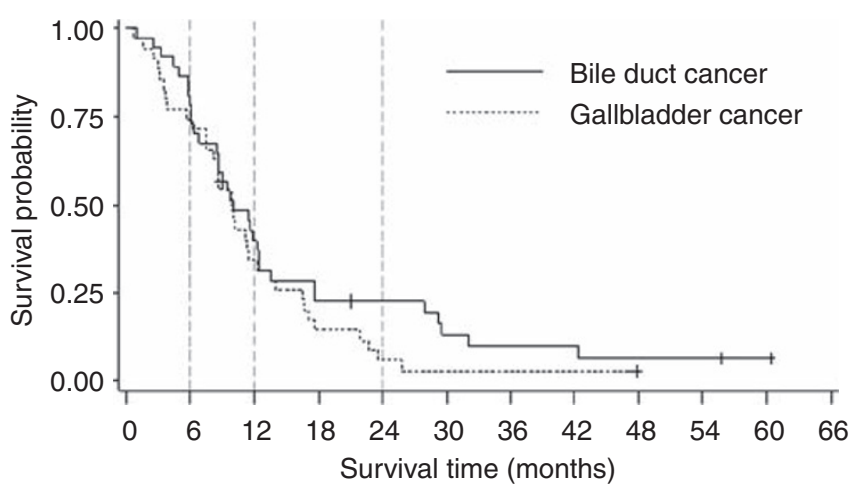

Figure 2 Overall survival (Kaplan-Meier) for patients with gallbladder cancer $(n=35)$ and bile duct cancer $(n=37)$ (ITT population). + , censored observations.

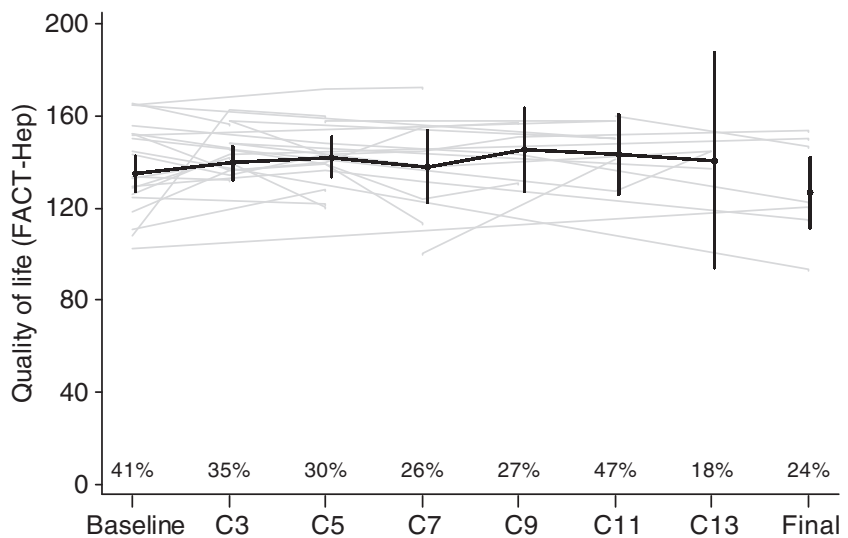

Figure 3 Quality of life (QoL), as measured by the FACT-Hep global score. Given are the individual (grey) and mean (black) global QoL scores (with 95\% confidence interval), as measured by the FACT-Hep questionnaire. High scores represent a high QoL with the maximum achievable value being 180, the minimum value 0 . Quality of life was evaluated at baseline, before cycles $3-13$ and at the final evaluation. Percentage values within the figure give the fraction of questionnaires divided by the number of patients still in the study at the respective time point. disease. There were no cardiac events or sudden deaths. Chemotherapy was administered as planned for $46.7 \%$ of scheduled treatments, was delayed for $17.9 \%$, required dose reduction for $18.9 \%$ or required both deferral and dose reduction for $13.4 \%$. One patient discontinued study therapy because of oxaliplatin-induced polyneuropathy. A total of 17 hospitalisations occurred during and in the first 30 days after chemotherapy in GBC patients. Among these, three were attributed to febrile infections, among which two were febrile neutropenia.

\section{DISCUSSION}

On the basis of favourable results of studies using two-drug combinations including gemcitabine, oxaliplatin and fluoropyrimidines (instead of 5-FU) (Alberts et al, 2005; Knox et al, 2005; Nehls et al, 2008), our trials were conducted to establish the efficacy and safety of the triplet GemFOx chemotherapy in patients with BDC and GBC. Taken together, these multicentre phase II studies, which include a total of 72 patients, represent one of the largest published series evaluating a single combination chemotherapy regimen in BTC. In addition, follow-up (up to 60 months) in this trial was longer than in most other studies. As results from randomised studies of chemotherapy in BTC are limited to two phase III trials $(n=54)$ (Rao et al, 2005; Valle et al, 2009) and a small number of phase II trials ( $n=22-86$ patients) (Kornek et al, 2004; Ducreux et al, 2005; Rao et al, 2005; Ciuleanu et al, 2007), treatment decisions in these tumours have to also consider the results of single-arm trials.

Both BDC and GBC are a clinically heterogeneous group of cancers. Increasing evidence that these differences are also seen at the molecular levels (Jarnagin et al, 2006) lends further support to the notion that 'gallbladder cancer is a different disease that needs individual trials' (Gallardo et al, 2005). Despite their heterogeneity, the majority of previously published trials include both BDC and GBC. In contrast, we evaluated - for the first time - the same combination chemotherapy regimen separately and with adequate power in patients with BDC and GBC. Although our trials are inevitably limited by the single-arm study design, they provide important and new clinical information regarding the efficacy and toxicity of the triplet chemotherapy combination under investigation.

Table 3 Most frequent grade III/IV adverse effects (NCI-CTC) observed in BDC and GBC trials

\begin{tabular}{|c|c|c|c|c|c|c|c|c|}
\hline & \multicolumn{4}{|c|}{ BDC } & \multicolumn{4}{|c|}{ GBC } \\
\hline & Grade I & Grade II & Grade III & Grade IV & Grade I & Grade II & Grade III & Grade IV \\
\hline Haemoglobin & $18(47.4)$ & $14(36.8)$ & $4(10.5)$ & $2(5.3)$ & $21(58.3)$ & $18(50.0)$ & $2(5.6)$ & $0(0)$ \\
\hline Leukocytes & $21(55.3)$ & $17(44.7)$ & $15(13.2)$ & $0(0)$ & $26(72.2)$ & $15(41.7)$ & $2(5.6)$ & | (2.8) \\
\hline Neutrophils/granulocytes & $15(39.5)$ & $12(31.6)$ & $9(23.7)$ & $5(13.2)$ & $18(50.0)$ & $13(36.1)$ & $10(27.8)$ & $2(5.6)$ \\
\hline Platelets & $21(55.3)$ & | | (28.9) & $3(7.9)$ & $4(10.5)$ & $16(44.4)$ & $15(41.7)$ & $10(27.8)$ & $3(8.3)$ \\
\hline Bilirubin & I (2.6) & $10(26.3)$ & $7(18.4)$ & $8(2||)$. & $2(5.6)$ & $10(27.8)$ & $5(13.9)$ & I (2.8) \\
\hline AST/ALT & $29(76.3)$ & $16(42.1)$ & $9(23.7)$ & $0(0)$ & $27(75.0)$ & $10(27.8)$ & $2(5.6)$ & $0(0)$ \\
\hline Alkaline phosphatase & $26(68.4)$ & $15(39.5)$ & $7(18.4)$ & $0(0)$ & $24(66.7)$ & $9(25.0)$ & $4(11.1)$ & $0(0)$ \\
\hline Nausea & $27(7||)$. & $12(31.6)$ & I (2.6) & $0(0)$ & $28(77.8)$ & $16(44.4)$ & $3(8.3)$ & $0(0)$ \\
\hline Diarrhoea & $15(39.5)$ & $2(5.3)$ & $2(5.3)$ & $0(0)$ & $13(36.1)$ & $6(16.7)$ & $0(0)$ & $0(0)$ \\
\hline Anorexia & $23(60.5)$ & $7(18.4)$ & $6(15.8)$ & $2(5.3)$ & $29(80.6)$ & $16(44.4)$ & $8(22.2)$ & | (2.8) \\
\hline Oedema & $5(13.2)$ & $8(2||)$. & $6(15.8)$ & $0(0)$ & $6(16.7)$ & $7(19.4)$ & $3(8.3)$ & $0(0)$ \\
\hline Dyspnea & $3(7.9)$ & $3(7.9)$ & $3(7.9)$ & I (2.6) & $3(8.3)$ & $5(13.9)$ & $3(8.3)$ & $2(5.6)$ \\
\hline Sensory neuropathy & $24(63.2)$ & $10(26.3)$ & $3(7.9)$ & $0(0)$ & $26(72.2)$ & $19(52.8)$ & $7(19.4)$ & $0(0)$ \\
\hline Fatigue & $18(47.4)$ & $27(71.1)$ & $3(7.9)$ & I (2.6) & $21(58.3)$ & $24(66.7)$ & $5(13.9)$ & | (2.8) \\
\hline Fever & II (28.9) & $12(31.6)$ & $2(5.3)$ & $0(0)$ & $9(25.0)$ & $10(27.8)$ & $3(8.3)$ & $0(0)$ \\
\hline Infection & $6(15.8)$ & II (28.9) & $3(7.9)$ & $0(0)$ & $5(13.9)$ & $13(36.1)$ & $3(8.3)$ & $0(0)$ \\
\hline Febrile neutropenia & $0(0)$ & $0(0)$ & I (2.6) & $0(0)$ & $0(0)$ & $0(0)$ & $2(5.6)$ & (0) \\
\hline
\end{tabular}

Abbreviations: $\mathrm{ALT}=$ alanine amino transaminase; $\mathrm{AST}=$ aspartate amino transaminase; $\mathrm{BDC}=$ bile duct cancer; $\mathrm{GBC}=$ gallbladder cancer. $\mathrm{Absolute}($ and relative, in \%) number of patients. 
In our BDC trial, although the objective response rate of $19 \%$ and the median overall survival of 10 months compare favourably with the results obtained with single-agent 5-FU or gemcitabine (Ducreux et al, 2005; Valle et al, 2009), the hypothesis that GemFOx might further improve on the results achieved with modern two-drug combinations (André et al, 2004, 2008; Alberts et al, 2005; Knox et al, 2005; Kim et al, 2006) was not confirmed. Nehls et al (2008) recently observed that adenocarcinoma of extrahepatic bile ducts, including Klatskin's tumours of the liver hilus, seems to have a better prognosis and response to chemotherapy than does intrahepatic cholangiocarcinoma. Furthermore, a differential expression of molecular targets such as HER-2 has been shown recently in intrahepatic and extrahepatic BDC (Yoshikawa et al, 2008). Therefore, possible variations in the relative proportions of intrahepatic and extrahepatic BDC might be a confounding factor in previously published studies, rendering conclusions regarding the efficacy of particular chemotherapy regimens difficult. Although we had insufficient data to retrospectively stratify our patients with respect to BDC subtype, and sound published epidemiological data are lacking, an unpublished retrospective 3-year (1 January 2006 to 31 December 2008) histopathological series of 839 unselected, consecutive cases of BDC in Germany suggests that $\sim 70 \%$ of these tumours are adenocarcinomas of the extrahepatic bile ducts (A Tannapfel, personal communication, January 2009). Future studies should address and clarify this issue by prospectively stratifying for the intrahepatic and extrahepatic type of BDC.

For GBC, owing to the even more limited data, the question of whether our triplet chemotherapy combination is superior to the single-agent or two-drug combination regimens tested in previous trials is more difficult to answer. Only 12 single-arm phase II and no phase III studies in patients with GBC were identified in a recent systematic search (Eckel and Schmid, 2007). Separate results for GBC were reported in 18 further studies with a mixed BTC population (F Eckel, personal communication, April 2008). We updated this search in October 2008 and identified only one additional abstract describing a prospective chemotherapy trial in GBC (Gallardo et al, 2008). Overall survival in the larger series $(\geqslant 40$ patients) was 5.7 (Chatni et al, 2008), 7.0 (Reyes-Vidal et al, 2003), 7.4 (Misra et al, 2005) and 9.0 months (Gallardo et al, 2008). Published results for GBC of all multicentre series, which included more than 20 patients, are available as Supplementary Online Material. Compared with these series, the median overall survival of 9.9 months and 1-year overall survival rate of $36 \%$ observed in our trial are encouraging. However, these results require confirmation in a randomised study.

Intensified chemotherapy using a three-drug combination might eventually allow for secondary resection of initially unresectable disease. In fact, none of the patients in our trials qualified for secondary resection, and we consider it unlikely that other currently available chemotherapy combinations will perform better. Thus, achieving secondary resectability appears to be an unrealistic goal for chemotherapy regimens available at present in patients with BTC.

The second major goal of our trials was to define the toxicity of this triplet chemotherapy in the two patient populations: Overall, the toxicity of the GemFOx combination chemotherapy in both patient populations was manageable. However, as expected, the rates of grade III and IV neutropenia (36.9 and $33.4 \%$ in patients with BDC and GBC), thrombopenia (18.4 and $36.1 \%$ in BDC and GBC) and anorexia (21 and $25.0 \%$ in BDC and GBC) in our study were increased when compared with the recently presented data for the gemcitabine/cisplatin combination in BTC (grade III/IV neutropenia $22.6 \%$, thrombopenia $8.2 \%$ and anorexia $1.9 \%$ ) (Valle et al, 2009). However, events such as febrile neutropenia (2.6 and $5.6 \%$ in BDC and GBC, respectively) or grade III/IV bleeding were low, and there were no treatment-related deaths. As expected, the risk of cholangitis was more important in patients with BDC compared with those with GBC. In contrast to our trial in pancreatic cancer (Wagner et al, 2007), the incidence of cardiovascular events was unremarkable.

In conclusion, a GemFOx triplet chemotherapy regimen is feasible in patients with advanced BTC, although the toxicity is increased compared with doublets. Although for patients with BDC the response rates and median overall survival in our trial do not exceed the results reported for the use of chemotherapy doublets, median overall survival for GBC compares favourably with most other published trials. However, the number of patients included in this trial is too small to draw definitive conclusions about the balance between benefit and toxicity of this three-drug combination chemotherapy regimen in BTC. Future drug development in BTC must account for the heterogeneity of BTC. The evolving understanding of the biology of these tumours confirms the existence of differences between GBC, as well as intrahepatic and extrahepatic BDC, at the molecular level. More importantly, it provides a basis for the rational use of targeted therapies that are currently under clinical evaluation. Whether tumour response is an appropriate primary end point for future clinical trials in biliary neoplasms is still open to question (Gores et al, 2004). Overall survival and patient-reported QoL have the advantage of being direct indicators of patient benefit.

\section{ACKNOWLEDGEMENTS}

These trials were supported in part by Lilly Deutschland $\mathrm{GmbH}$ (Germany) and Sanofi-Synthelabo (Paris, France) and by the Coordinating Centre for Clinical Trials, Halle (supported by the German Ministry for Education and Research, Grant number BMBF/FKZ: 01GH0105 KKS Halle). We express our thanks to Lena Minning and Mareike Kunze (Institute of Medical Epidemiology, Biostatistics, and Informatics, Martin-Luther-University HalleWittenberg, Halle, Germany) for data management, as well as to PD Dr J Barth (Hospital 'Bergmannstrost', Halle (Saale)) Dr D Arnold (Department of Medicine IV, University Hospital, Halle (Saale)) and Professor Dr Busse (Städtisches Krankenhaus Martha-Maria, Halle (Saale)) for the inclusion of two patients each; Professor W Schepp (Krankenhaus München-Bogenhausen) for the inclusion of three patients; PD Dr C Ridwelski (Städtisches Klinikum Dessau) for the inclusion of one patient; and Drs Hahnfeld and Krombholz (Jena) for the inclusion of four patients. In addition, we thank Professor Heinz-Josef Lenz, UCLA for helpful discussion of the manuscript.

Supplementary Information accompanies the paper on British Journal of Cancer website (http://www.nature.com/bjc)

\section{REFERENCES}

Alberts SR, Al Khatib H, Mahoney MR, Burgart L, Cera PJ, Flynn PJ, Finch TR, Levitt R, Windschitl HE, Knost JA, Tschetter LK (2005) Gemcitabine, 5-fluorouracil, and leucovorin in advanced biliary tract and gallbladder carcinoma: a North Central Cancer Treatment Group phase II trial. Cancer 103: $111-118$

André T, Reyes-Vidal JM, Fartoux L, Ross P, Leslie M, Rosmorduc O, Clemens MR, Louvet C, Perez N, Mehmud F, Scheithauer W (2008)
Gemcitabine and oxaliplatin in advanced biliary tract carcinoma: a phase II study. Br J Cancer 99: 862-867

André T, Tournigand C, Rosmorduc O, Provent S, Maindrault-Goebel F, Avenin D, Selle F, Paye F, Hannoun L, Houry S, Gayet B, Lotz JP, de Gramont A, Louvet C (2004) Gemcitabine combined with oxaliplatin (GEMOX) in advanced biliary tract adenocarcinoma: a GERCOR study. Ann Oncol 15: 1339-1343 
Chatni SS, Sainani RS, Mehta SA, Mohandas KM (2008) Infusion chemotherapy with cisplatinum and fluorouracil in the treatment of locally-advanced and metastatic gallbladder cancer. J Cancer Res Ther 4: 151-155

Ciuleanu T, Diculescu M, Hoepffner NM, Trojan J, Sailer V, Zalupski M, Herrmann T, Roth A, Chick J, Brock K, Albert D, Philip PA (2007) A randomised phase II study of OSI-7904L vs 5-fluorouracil (FU)/leucovorin (LV) as first-line treatment in patients with advanced biliary cancers. Invest New Drugs 25: 385-390

Cunningham D, Chau I, Stocken C, Davies C, Dunn J, Valle J, Smith D, steward W, Harper P, Neoptolemos J (2005) Phase III randomized comparison of gemcitabine (gem) vs gemcitabine plus capecitabine (Gem-CAP) in patients with advanced pancreatic cancer. Eur J Cancer 3(suppl 4); abstract no. PS 11

Ducreux M, Van Cutsem E, Van Laethem JL, Gress TM, Jeziorski K, Rougier P, Wagener T, Anak O, Baron B, Nordlinger B (2005) A randomised phase II trial of weekly high-dose 5-fluorouracil with and without folinic acid and cisplatin in patients with advanced biliary tract carcinoma: results of the 40955 EORTC trial. Eur J Cancer 41: 398-403

Eckel F, Schmid RM (2007) Chemotherapy in advanced biliary tract carcinoma: a pooled analysis of clinical trials. Br J Cancer 96: 896-902

Faivre S, Raymond E, Woynarowski JM, Cvitkovic E (1999) Supraadditive effect of $2^{\prime}, 2^{\prime}$-difluorodeoxycytidine (gemcitabine) in combination with oxaliplatin in human cancer cell lines. Cancer Chemother Pharmacol 44: 117-123

Gallardo J, Rubio B, Ahumada M, Barajas O, Villanueva L, Diaz J, De Aretxabala X, Reyes J (2008) Therapy for advanced gallbladder cancer: improving survival. ASCO Annu Meet Proc 26; abstract no. 15566

Gallardo J, Rubio B, Villanueva L, Barajas O (2005) Gallbladder cancer, a different disease that needs individual trials. J Clin Oncol 23: $7753-7754$

Glimelius B, Hoffman K, Sjoden PO, Jacobsson G, Sellstrom H, Enander LK, Linne T, Svensson C (1996) Chemotherapy improves survival and quality of life in advanced pancreatic and biliary cancer. Ann Oncol 7: 593-600

Gores GJ, Baskin-Bey ES, Baron TH, Alberts SR (2004) Treatment endpoints for advanced cholangiocarcinoma. Nat Clin Pract Gastroenterol Hepatol 1: 4-5

Heffernan N, Cella D, Webster K, Odom L, Martone M, Passik S, Bookbinder M, Fong Y, Jarnagin W, Blumgart L (2002) Measuring health-related quality of life in patients with hepatobiliary cancers: the functional assessment of cancer therapy-hepatobiliary questionnaire. J Clin Oncol 20: 2229-2239

Hezel AF, Zhu AX (2008) Systemic therapy for biliary tract cancers. Oncologist 13: 415-423

Jarnagin WR, Klimstra DS, Hezel M, Gonen M, Fong Y, Roggin K, Cymes K, DeMatteo RP, D'Angelica M, Blumgart LH, Singh B (2006) Differential cell cycle-regulatory protein expression in biliary tract adenocarcinoma: correlation with anatomic site, pathologic variables, and clinical outcome. J Clin Oncol 24: $1152-1160$

Kaplan EL, Meier P (1958) Nonparametric-estimation from incomplete observations. J Am Stat Assoc 53: 457-481

Kim ST, Park JO, Lee J, Lee KT, Lee JK, Choi SH, Heo JS, Park YS, Kang WK, Park K (2006) A phase II study of gemcitabine and cisplatin in advanced biliary tract cancer. Cancer 106: $1339-1346$

Knox JJ, Hedley D, Oza A, Feld R, Siu LL, Chen E, Nematollahi M, Pond GR, Zhang J, Moore MJ (2005) Combining gemcitabine and capecitabine in patients with advanced biliary cancer: a phase II trial. J Clin Oncol 23: 2332-2338

Kobayashi K, Tsuji A, Morita S, Horimi T, Shirasaka T, Kanematsu T (2006) A phase II study of LFP therapy (5-FU (5-fluorouracil) continuous infusion (CVI) and low-dose consecutive (Cisplatin) CDDP) in advanced biliary tract carcinoma. BMC Cancer 6: 121

Kornek GV, Schuell B, Laengle F, Gruenberger T, Penz M, Karall K, Depisch D, Lang F, Scheithauer W (2004) Mitomycin C in combination with capecitabine or biweekly high-dose gemcitabine in patients with advanced biliary tract cancer: a randomised phase II trial. Ann Oncol 15: $478-483$

Miller AB, Hoogstraten B, Staquet M, Winkler A (1981) Reporting results of cancer treatment. Cancer 47: 207-214

Misra S, Chaturvedi A, Misra NC (2005) Gemcitabine(G) plus cisplatin (C) in advanced carcinoma gallbladder - a large single center experience. ASCO Annu Meet Proc 23; abstract no. 4136

Nehls O, Oettle H, Hartmann JT, Hofheinz RD, Hass HG, Horger MS, Koppenhofer U, Hochhaus A, Stieler J, Trojan J, Gregor M, Klump B (2008) Capecitabine plus oxaliplatin as first-line treatment in patients with advanced biliary system adenocarcinoma: a prospective multicentre phase II trial. Br J Cancer 98: 309-315

Patt YZ, Hassan MM, Lozano RD, Waugh KA, Hoque AM, Frome AI, Lahoti S, Ellis L, Vauthey JN, Curley SA, Schnirer II, Raijman I (2001) Phase II trial of cisplatin, interferon alpha-2b, doxorubicin, and 5-fluorouracil for biliary tract cancer. Clin Cancer Res 7: 3375-3380

Peters GJ, Bergman AM, Ruiz van Haperen VW, Veerman G, Kuiper CM, Braakhuis BJ (1995) Interaction between cisplatin and gemcitabine in vitro and in vivo. Semin Oncol 22: $72-79$

Randi G, Malvezzi M, Levi F, Ferlay J, Negri E, Francesci S, La Veccia C (2009) Epidemiology of biliary tract cancers: an update. Ann Oncol 20: $146-159$

Rao S, Cunningham D, Hawkins RE, Hill ME, Smith D, Daniel F, Ross PJ, Oates J, Norman AR (2005) Phase III study of 5FU, etoposide and leucovorin (FELV) compared to epirubicin, cisplatin and 5FU (ECF) in previously untreated patients with advanced biliary cancer. $\mathrm{Br} J$ Cancer 92: $1650-1654$

Reyes-Vidal J, Gallardo J, Yanez E, Rosas J, Palma M, Cerda B, Loredo E, Del Castillo C, Arriagada R, Quijada I (2003) Gemcitabine (G) and cisplatin (C) in the treatment of patients (pts) with unresectable or metastatic gallbladder cancer: results of the phase II GOCCHI study 2000-13. Proc Am Soc Clin Oncol 22; abstract no. 1095

Riess H, Helm A, Niedergethmann M, Schmidt-Wolf J, Moik M, Hammer C, Zippel K, Weigang-Köhler K, Stauch M, Oettle H (2005) A randomized, prospective, multicenter phase III trial of gemcitabine, 5-fluorouracil (5-FU), folinic acid $v s$ gemcitabine alone in patients with advanced pancreatic cancer. Proc Am Soc Clin Oncol 23; abstract no. 4009

Sanz-Altamira PM, Ferrante K, Jenkins RL, Lewis WD, Huberman MS, Stuart KE (1998) A phase II trial of 5-fluorouracil, leucovorin, and carboplatin in patients with unresectable biliary tree carcinoma. Cancer 82: $2321-2325$

Valle JW, Wasan H, Palmer DD, Cunningham D, Anthoney A, Maraveyas A, Hughes SK, Roughton M, Bridgewater J (2009) Gemcitabine with or without cisplatin in patients (pts) with advanced or metastatic biliary tract cancer (ABC): Results of a multicenter, randomized phase III trial (the UK ABC-02 trial). ASCO Annu Meet Proc 27; abstract no. 4503

Wagner AD, Buechner-Steudel P, Wein A, Schmalenberg H, Lindig U, Moehler M, Behrens R, Kleber G, Kuss O, Fleig WE (2007) Gemcitabine, oxaliplatin and weekly high-dose 5 -FU as 24 -h infusion in chemonaive patients with advanced or metastatic pancreatic adenocarcinoma: a multicenter phase II trial of the Arbeitsgemeinschaft Internistische Onkologie (AIO). Ann Oncol 18: 82-87

Yonemoto N, Furuse J, Okusaka T, Yamao K, Funakoshi A, Ohkawa S, Boku N, Tanaka K, Nagase M, Saisho H, Sato T (2007) A multi-center retrospective analysis of survival benefits of chemotherapy for unresectable biliary tract cancer. Jpn J Clin Oncol 37: 843-851

Yoshikawa D, Ojima H, Iwasaki M, Hiraoka N, Kosuge $T$, Kasai S, Hirohashi S, Shibata T (2008) Clinicopathological and prognostic significance of EGFR, VEGF, and HER2 expression in cholangiocarcinoma. Br J Cancer 98: $418-425$ 